\title{
EFFECT OF THERMAL ANNEALING ON OPTICAL PROPERTIES OF IMPLANTED GaAs
}

\author{
M. KULIK ${ }^{a}$, F.F. Komarov ${ }^{b}$ AND D. MĄCZKa ${ }^{a}$ \\ ${ }^{a}$ Institute of Physics, Maria Curie-Skłodowska University \\ Pl. Marii Curie-Skłodowskiej 1, 20-031 Lublin, Poland \\ ${ }^{b}$ Institute of Physics, State University, Minsk, Republic of Byelorussia
}

\begin{abstract}
GaAs samples doped with indium atoms by ion implantation and thermal annealed were studied using a channelling method, Rutherford backscattering, and an ellipsometry. From these measurements it was observed that the layer implanted with $3 \times 10^{16} \mathrm{~cm}^{-2}$ indium dose was totally damaged and its optical properties, namely a refraction index $n$ and an extinction coefficient $k$, corresponded to the amorphous material. Subsequent isobaric heating of the implanted samples resulted in recovery of the crystalline structures with simultaneous change of the $n$ and $k$ index values.
\end{abstract}

PACS numbers: 07.60.Fs, 61.72.Vv, 78.20.-e

\section{Introduction}

Ion implantation is an important process in semiconductor integrated circuit technology [1]. Therefore, in recent years intensive comparative studies were carried out on the effect of ion implantation on optical properties of $A_{3} B_{5}$ compounds [2-5]. In these investigations the channelling techniques, the ellipsometry, and the measurements of light transmission near the fundamental absorption edge were used. They resulted in obtaining information about the influence of radiation damages induced by active and electrically neutral impurities on change of the refraction index $n[4,5]$.

The $\operatorname{In}_{x} \mathrm{Ga}_{(1-x)}$ As compound is of importance from the point of view of optoelectronics. Studies were carried out in the recent years on obtaining a layer of this compound by implanting indium ions into GaAs and its thermal annealing [6]. The authors of that paper used the rapid thermal annealing (RTA) technique and Raman spectroscopy. The measurements have shown that a heating at $800^{\circ} \mathrm{C}$ gave satisfactory results in obtaining a stable ternary compound.

The present work is a continuation of the studies [6] and its purpose is to investigate the influence of ion implantation and isobaric thermal heating on the crystalline structure and optical properties of implanted layers. 


\section{Experiment}

In a present study the authors used semi-insulating undoped GaAs single crystals of a (100) orientation. Before ion bombardment the surfaces of all samples were chemically cleaned in bromomethanol solution to remove all impurities adsorbed from air [7]. Then $3 \times 10^{16} \mathrm{~cm}^{-2}$ indium dose was implanted into the samples. The energy of the bombarding ions was $200 \mathrm{keV}$, while the density of the ion current amounted $1.44 \mu \mathrm{A} \mathrm{cm}^{-2}$. Ion implantation proceeds at room temperature and the angle between the sample planes and the incidence of the ion beam was $82^{\circ}$. The above condition guaranteed that the effects of ion channelling and thermal heating did not occur during implantation. Thus prepared samples were covered with a protective $0.5 \mu \mathrm{m}$ thick $\mathrm{SiO}_{2}$ layer. In the following step the implanted samples were annealed in the temperature range from $400^{\circ} \mathrm{C}$ to $950^{\circ} \mathrm{C}$. The annealing was conducted at a constant pressure of an Ar gas flow. The heating time was $0.5 \mathrm{~h}$. For the samples measured by the methods of channelling and Rutherford. backscattering (RBS) the recovery of the crystalline structure and indium diffusion during postimplantation annealing were examined respectively. RBS spectra were measured using the Van de Graaff accelerator AN-2500 (High Voltage Engineering Comp.) with a spectrometer (ORTEC). The energy spread of the ions generated by the accelerator was less than $15 \mathrm{keV}$. The backscattering angle was $170^{\circ}$. The $2.0 \mathrm{MeV}$ He ion beam was used for analysis.

For the implanted GaAs layers optical parameters were also determined on the basis of ellipsometric studies and the multiple-angle-of-incidence (MAI) method [8]. An automatic ellipsometer EL11D was used for one light wavelength $\lambda=632.8 \mathrm{~nm}$. The incidence angle of the light beam was changed in the interval from 60 to 78 degrees. Using such geometry of the ellipsometric measurements it was possible to obtain the maximal sensitivity and accuracy in calculations of optical parameters. For calculation of the refraction $n$ and extinction $k$ indices of unimplanted GaAs samples the three-phase model (an ambient, a thin plano-parallel layer and a homogeneous isotropic substrate) was used. In the case of the implanted and heated samples we utilized the four-phase model. In all calculations it was assumed that the imptanted layers were homogeneous.

\section{Results and discussion}

From the channelling measurements it was found that implantation with In ions of $200 \mathrm{keV}$ energy and $3 \times 10^{16} \mathrm{~cm}^{-2}$ dose induced in GaAs samples the formation of an amorphous layer. However, thermal annealing at $t=950^{\circ} \mathrm{C}$ leads to almost complete recovery of the crystalline structure of the material (Fig. 1). For the samples annealed at a lower temperature the presence of a layer was found in which a partial disorder was observed.

The concentration distributions of indium atoms in the implanted layer was determined on the basic of the RBS measurements (Fig. 2). The evaluation of the In concentration distributions upon the amnealing temperature is shown in Fig. 3. From this figure it follows that indium atoms introduced into GaAs diffuse in two directions - towards the surface and deep into the sample. Indium diffusion to the surface of irradiated samples is preferable in the material heated at the temperature below $600^{\circ} \mathrm{C}$. 


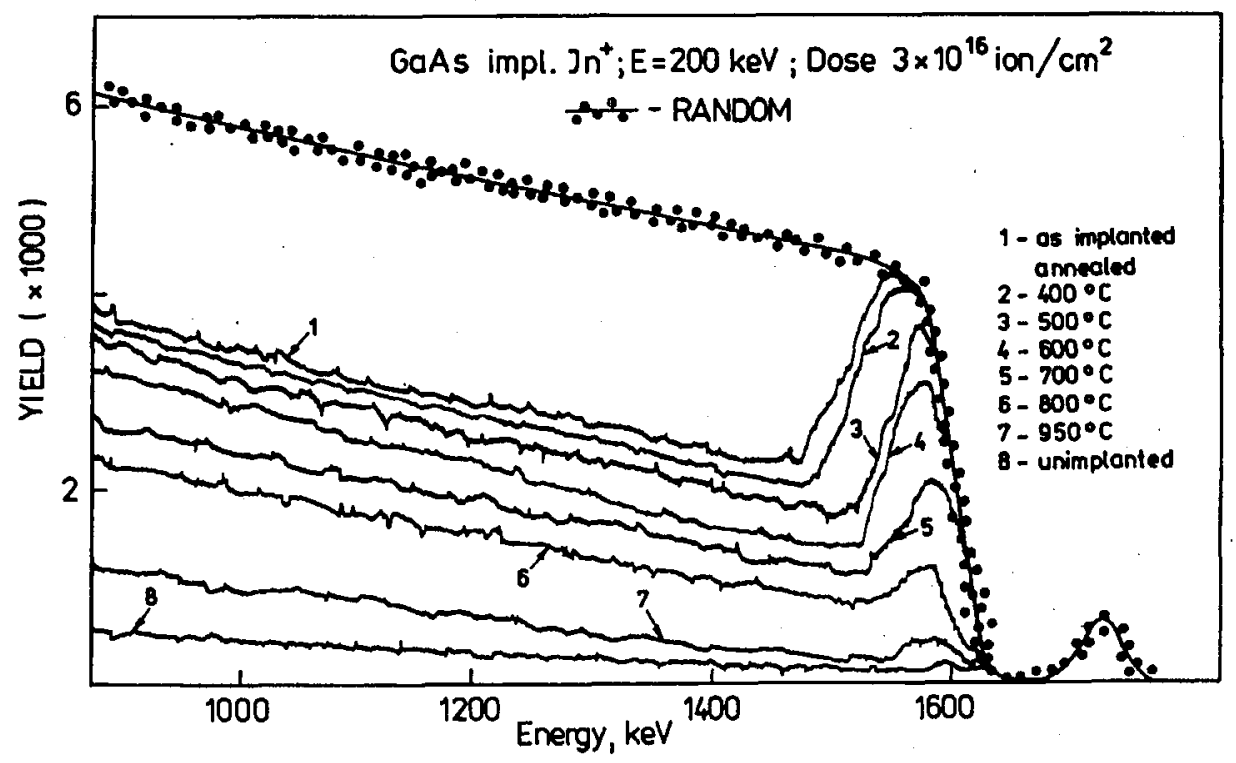

Fig. 1. RBS and channelling spectra of GaAs implanted with $200 \mathrm{keV} \mathrm{In}^{+}$; before and after thermal annealing.

For the samples heated at the temperature above $600-700^{\circ} \mathrm{C}$ the depth distribution of implanted atoms is constant and the theoretical depth $R_{p}+\Delta R_{p}=$ $88.2 \mathrm{~nm}$ was calculated with the TRIM code [9] (Fig. 3).

Using the ellipsometric method the refraction index $n$ and the extinction coefficient $k$ of implanted samples were determined. The results are given in Table. As it appears from the Table, the optical constants of the doped and unheated GaAs layer correspond to the values of amorphous material $(n=4.35, k=1.06)$ [10]. The existence of an amorphous layer has been also confirmed by the channelling measurements (Fig. 1). Moreover, it was found that with the increased annealing temperature, the values of the refraction inclex $n$ and the extinction coefficient $k$ of. the implanted layer decrease. This effect can be attributed to two processes. The first one is connected with the recovery of the crystalline structure of the damaged layer; it is mainly responsible for the decrease in the extinction index. The other process consists in substitution of the indium atoms into sites of knocked-out gallium atoms. As a result of the latter, the formation of compounds of InAs and $\operatorname{In}_{x} \mathrm{Ga}_{(1-x)}$ As type can be expected. For such supposition the $x$ value can be calculated. From the RBS measurements, this value reached about 0.051 , while from the calculation on the basis of the implanted dose $x \approx 0.063$. The consequence of the formation of the ternary compound in the implanted layers of GaAs samples is the increase in the refraction and extinction indices above those corresponding to the initial material (Table). The obtained results confirm the expectations and results of the paper [6]. 


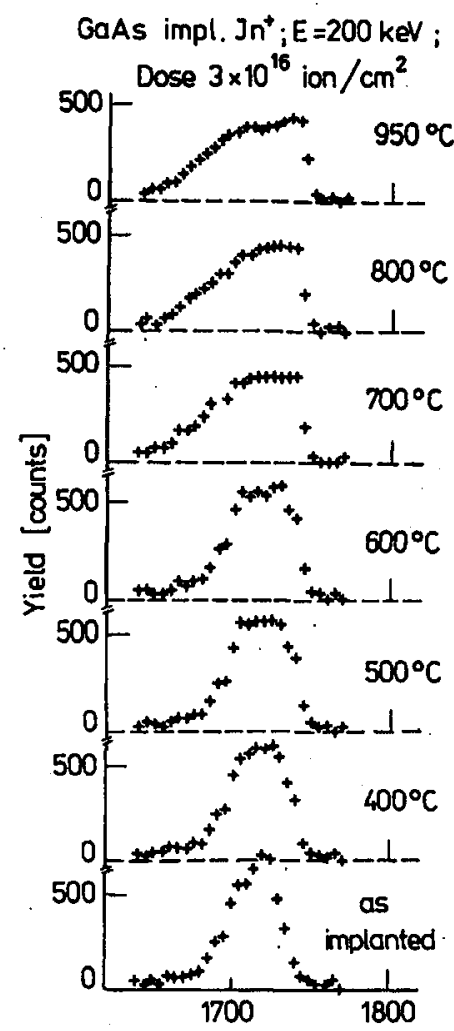

Fig. 2 Energy [keV]

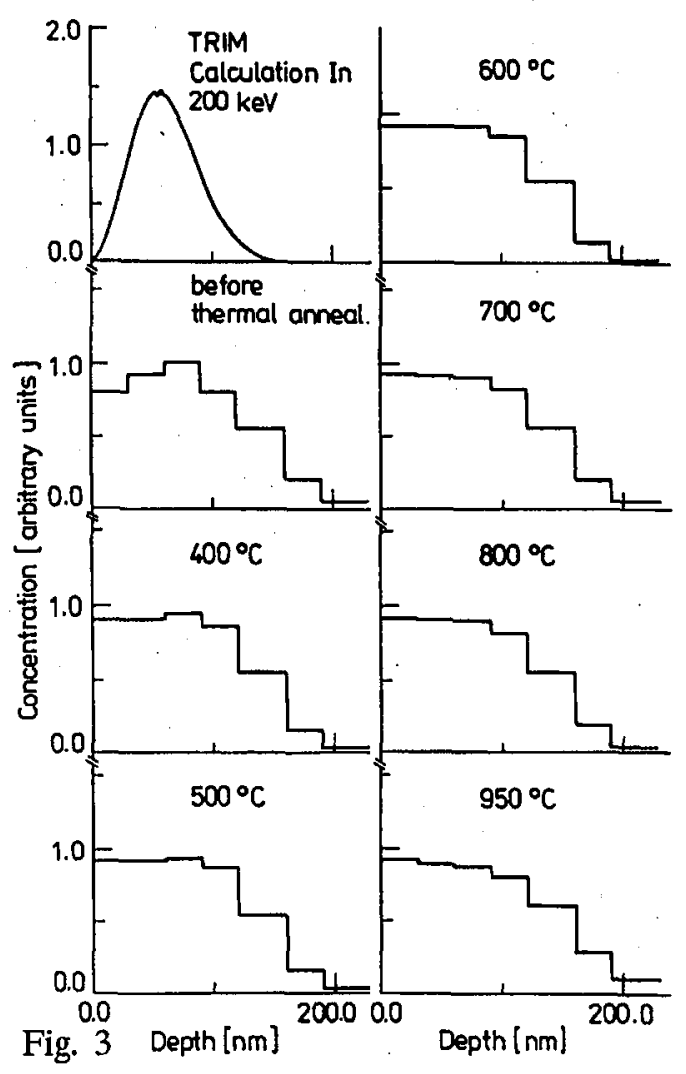

Fig. 3 Depth $[\mathrm{nm}]$

Fig. 2. The part of the RBS spectra of GaAs implanted with $\mathrm{In}^{+}$in the range of $\mathrm{He}^{+}$ ions from $1640 \mathrm{keV}$ to $1820 \mathrm{keV}$ (before and after thermal annealing).

Fig. 3 Depth distributions of indium atoms in GaAs implanted with $200 \mathrm{keV} \mathrm{In}$; calculated with the help of TRIM code and obtained from.the RBS measurements: (before and after thermal annealing).

TABLE

Optical constants of GaAs implanted with In ions determined with the help of ellipsometric measurements. The errors of the refraction $n$ and the extinction $k$ indices are less than \pm 0.008 and \pm 0.010 , respectively.

\begin{tabular}{c|c|c|c|c|c|c|c|c}
\hline \hline & unimpl. & as & \multicolumn{6}{|c}{ implanted and annealed } \\
\cline { 4 - 8 } & & impl. & \multicolumn{5}{|c}{$T$ annealing $\left[{ }^{\circ} \mathrm{C}\right]$} \\
\cline { 3 - 8 } & & 400 & 500 & 600 & 700 & 800 & 950 \\
\hline$n$ & 3.866 & 4.320 & 4.102 & 4.001 & 3.992 & 3.947 & 3.926 & 3.901 \\
$k$ & 0.257 & 1.081 & 1.004 & 0.895 & 0.802 & 0.628 & 0.471 & 0.311
\end{tabular}




\section{Conclusions}

From the presented studies it was found that the stable layers enriched with indium can be obtained by ion implantation and isobaric thermal annealing above $950^{\circ} \mathrm{C}$. These layers are ternary $\operatorname{In}_{x} \mathrm{Ga}_{(1-x)}$ As compounds, where $x \approx 0.051 \div 0.063$.

\section{Acknowledgments}

The authors would like to acknowledge J. Liśkiewicz for the implantation of GaAs samples.

\section{References}

[1] T. Lohner, E. Kotai, N.Q. Khanh, Z. Toth, M. Fried, K. Vedam, N.V. Nguyen, L.J. Hanekamp, A. van Silfhout, Nucl. Instrum. Methods B 85, 335 (1994).

[2] M. Fried, T. Lohner, E. Jaroli, Gy. Vizkelethy, G. Mezey, J. Gyulai, M. Somogyi, H. Kerkow, Thin Solid Films 116, 191 (1984).

[3] T. Lohner, Z. Toth, M. Fried, N.Q. Khanh, Gen Qing Yang Lin Chen Lu, Shichang Zou, L.J. Henekamp, A. van Silfhout, J. Gyulai, Nucl. Instrum. Methods B 85, 524 (1994).

[4] E. Wendler, M. Kulik, W. Wesch, T. Bachmann, Phys. Status Solidi A 126, K105 (1991).

[5] W. Wesch, E. Wendler, T. Bachmann, O. Herre, Nucl. Instrum. Methods B 95, 290 (1995).

[6] A.N. Akimov, L.A. Vlasukova, F.F. Komarov, M. Kulik, J. Appl. Spectrosc. 59, 96 (1993).

[7] D.E. Aspnes, A.A. Studna, Phys. Rev. B 27, 985 (1983).

[8] R.M.A. Azzam, N.M. Bashara, Ellipsometry and Polarized Light, North Holland, Amsterdam 1977.

[9] J.F. Ziegler, J.P. Biersak, V. Litmark, The Stopping Range of Ions in Matter, Pergamon Press, New. York 1985.

[10] M. Kulik, K. Jezierski, Acta Phys. Pol. A 75, 211 (1989). 